(C) 2021, The Authors. Published by Elsevier Inc. and Fass Inc. on behalf of the American Dairy Science Association ${ }^{\circledR}$. This is an open access article under the CC BY-NC-ND license (http://creativecommons.org/licenses/by-nc-nd/4.0/).

\title{
Effect of pentasodium triphosphate concentration on physicochemical properties, microstructure, and formation of casein fibrils in model processed cheese
}

\author{
Almut H. Vollmer, ${ }^{1 *} \odot$ Ingrun Kieferle, ${ }^{2} \odot$ Alexandra Pusl, ${ }^{2}$ and Ulrich Kulozik ${ }^{2} \odot$ \\ ${ }^{1}$ Department of Nutrition, Dietetics, and Food Sciences, Utah State University, Logan 84322 \\ ${ }^{2}$ Chair of Food and Bioprocess Engineering, TUM School of Life Sciences, Technical University of Munich, 85354 Freising, Germany
}

\begin{abstract}
The effects of varying the concentration of pentasodium triphosphate (PP) emulsifying salt $[0,0.6,1.2,1.5$, and $1.8 \%$, plus $0.9 \%$ of a mixture of citric acid (CA) and disodium phosphate (DSP) to adjust cheese $\mathrm{pH}$ to 5.85] on rheological, textural, physicochemical, and microstructural properties were studied in a processed cheese model system containing $\sim 20 \%$ micellar casein concentrate, $\sim 20 \%$ sunflower oil, and $\sim 59 \%$ water. Special emphasis was placed on the unique casein fibrils recently described in a comparable processed cheese model system. Our results show that during processing $\left(90^{\circ} \mathrm{C}, 17.37 \mathrm{rpm}\right.$ over $\left.270 \mathrm{~min}\right)$ the apparent viscosity increased more and faster for formulations containing higher concentrations of PP, in analogy to the so-called creaming reaction, a general thickening of the molten cheese mass with prolonged processing. We found that $1.2 \%$ PP (plus $0.9 \%$ CA-DSP) appeared to be the threshold for the creaming reaction to take place. With increasing PP concentrations, cheese hardness increased in a sigmoidal fashion, and insoluble (protein-bound) calcium concentration decreased exponentially. Light micrographs of samples taken at the end of processing indicated initially large and dense casein aggregates within the matrix that disappeared with higher levels of $\mathrm{PP}$, in parallel with the development of a finer emulsion. With transmission electron microscopy analysis on the same samples, the highly complex restructuring of the casein matrix was evident; casein fibrils had formed de novo at the periphery of the loosening casein aggregates. With higher levels of PP, amorphous areas were observed in place of the dense casein aggregates that appeared progressively void of protein, whereas fibril concentration increased throughout the rest of the matrix. Fibrils progressively attached to the surface of fat globules, thereby emulsifying them. Reverse-phase
\end{abstract}

Received April 19, 2021.

Accepted June 28, 2021

*Corresponding author: almut.vollmer@usu.edu
HPLC analysis of insoluble and soluble fractions indicated $\kappa$-casein to be the most likely constituent of the newly formed fibrils. The results of this study suggest that PP induced a concentration-dependent dissociation of caseins (through increased calcium chelation) and further led to their spatial separation. In essence, their chaperone activity was hindered, which resulted in amorphous aggregation on the one hand and fibril formation on the other.

Key words: amyloid fibrils, emulsifying salt, proteinprotein interactions, transmission electron microscopy

\section{INTRODUCTION}

Processed cheese is a dairy product that has garnered widespread attention since its creation more than one hundred years ago, owing to its numerous end-use applications such as loaves, slices, shreds, spreads, and sauces, or as an ingredient in various food preparations. Processed cheese is principally manufactured by blending comminuted natural cheese, water, and emulsifying salts, followed by cooking at high heat under constant shear to form a homogeneous molten mass, which is then packaged and cooled (Zehren and Nussbaum, 1992). The use of other protein and fat sources in processed cheese analogs and the addition of flavors has led to an even wider array of possibilities (reviewed in Kapoor and Metzger, 2008).

An integral part of the manufacture of processed cheese is the use of emulsifying or melting salts; sodium orthophosphates, polyphosphates, and citrates are the most common (Caric et al., 1985). Without them, the homogenized cheese mass would quickly become unstable, resulting in phase separation. Contrary to their name, emulsifying salts are not emulsifiers, at least not in the strict sense, as they are not surface-active compounds. However, they increase the emulsifying properties of caseins, the main dairy protein in processed cheese, by interacting with calcium and thus disrupting the calcium phosphate $(\mathbf{C a P})$ nanocluster complexes (also known as colloidal calcium phosphate), which 
stabilize casein micelles and the para-casein matrix. This interaction has often been described as calcium sequestration or calcium chelation, although the exact mode of action has not been elucidated; it appears to be different for different melting salts (Mizuno and Lucey, 2005). The dissociated and hydrated caseins are then assumed to interact via their hydrophobic sites with the fat phase, thereby emulsifying it. Additionally, emulsifying salts affect the $\mathrm{pH}$ of the cheese mass, decreasing or increasing it depending on the type and concentration of salt used (Kapoor and Metzger, 2008). Emulsifying salts can further be distinguished by their affinity to sequester calcium, arguably their most important function, with polyphosphates typically showing the highest affinity (Caric et al., 1985).

Ingredients and their formulations (including type and concentration of the aforementioned melting salts) and processing parameters (i.e., processing temperature and time, mixing speed, rate of cooling after manufacture) largely affect textural and functional attributes of processed cheese (reviewed in Kapoor and Metzger, 2008, and Masotti et al., 2018). In this regard, the phenomenon of the so-called creaming reaction, defined as a general thickening of the molten cheese mass during and even after processing (Zehren and Nussbaum, 1992), is important, as it increases the viscosity and firmness of the final product (Shirashoji et al., 2006; Chen and Liu, 2012). It should be noted that this reaction is not related to creaming of fat droplets in unhomogenized milk. The magnitude of the creaming reaction in processed cheese heavily depends on compositional and processing conditions (Röck, 2010; Fu et al., 2018; Lenze et al., 2019). In a recent study, we investigated the underlying mechanisms leading to this viscosity increase, using high-resolution transmission electron microscopy, and found that it was mainly related to a restructuring of the casein matrix (Vollmer et al., 2021). This had already been postulated by others (Lee et al., 2003; Kawasaki, 2008; Heertje, 2014). However, our study went further, and we showed a timedependent separation of the casein matrix into fibrillogenic and nonfibrillogenic fractions with subsequent formation and higher-order structuring of unique fibrils and their increasing interaction with the fat phase. The question as to the nature of these unique fibrils was not addressed.

Fibril-like structures have been reported for "hardtype" processed cheese but not for "soft-type" processed cheese, the difference being the composition and concentration of the melting salts used (Kimura and Taneya, 1975; Taneya et al., 1980; Tamime et al., 1990). Fibril formation is uncommon in liquid milk, although some evidence suggests the possible existence of very short
$(7-10 \mathrm{~nm})$ fibril-like structures in bovine casein micelles (Lencki, 2007). Amyloid fibril formation has been documented for $\kappa$-CN (Farrell et al., 2003; Thorn et al., 2005; Leonil et al., 2008), for $\alpha_{S_{2}}-\mathrm{CN}$ (Thorn et al., 2008 ), and to some extent for isolated $\beta-\mathrm{CN}$ (Pan and Zhong, 2015). The latter, however, formed fibrils only under nonphysiological conditions at $\mathrm{pH} 2$ and $90^{\circ} \mathrm{C}$ over $30 \mathrm{~h}$. Fibril formation has not been reported for isolated $\alpha_{S_{1}}-\mathrm{CN}$, although this is theoretically possible based on the inherently disordered state of the protein, with significant poly-L proline type II helix formation (Carver and Holt, 2019). Interestingly, the in vitro formation of amyloid fibrils by $\kappa-\mathrm{CN}$ was suppressed by $\alpha_{\mathrm{S}^{-}} \mathrm{CN}\left(\alpha_{\mathrm{S} 1}-\mathrm{CN}\right.$ plus $\left.\alpha_{\mathrm{S}^{-}} \mathrm{CN}\right)$, or $\beta-\mathrm{CN}$, when offered in excess molarities, and $\alpha_{\mathrm{S} 1}-\mathrm{CN}$ but not $\beta$-CN suppressed fibril formation by $\alpha_{\mathrm{S}_{2}}$-CN (Thorn et al., 2008; Treweek et al., 2011). This molecular chaperone activity explains how caseins can be protected from dysfunctional aggregation when they associate to form the native casein micelle in milk (Carver and Holt, 2019). However, there are apparent limitations to this protective mechanism, as indicated by the existence of corpora amylacea (pathological calcifications in the alveoli of mammary glands of lactating cows). Characteristic for these aberrant deposits is their amyloid fibril-like morphology in certain parts and the presence of $\alpha_{S_{2}}-\mathrm{CN}$, among other proteins (Brooker, 1978; Claudon et al., 1998).

The aim of our study was to investigate the influence of pentasodium triphosphate (PP), an emulsifying salt with high calcium affinity, on the formation of fibrils in a high-moisture ( $\sim 59 \%$ moisture) processed cheese model system where creaming takes place after extended heating and mixing, and how fibril formation is related to the examined physicochemical and textural properties. We further attempted to identify the subunits involved in the formation of these unique fibrils.

\section{MATERIALS AND METHODS}

\section{Composition of the Processed Cheese Model System}

The formulation of the model processed cheese can be found in Table 1. The protein source consisted of micellar casein concentrate (MCC) powder and was manufactured according to Dumpler (2018) at the Technical University of Munich, Germany. Pasteurized skim milk $(300 \mathrm{~L})$ was microfiltered at 50 to $52^{\circ} \mathrm{C}$ using $1.4-\mu \mathrm{m}$ ceramic gradient membranes (TAMI Isoflux Sunflower, TAMI Germany GmbH), followed by 8 diafiltration steps with lactose-free simulated milk ultrafiltrate to remove whey proteins, lactose, and nonprotein nitrogen components. After diafiltration, the MF retentate was 
Table 1. Formulation of the model processed cheese; $\mathrm{pH}$ values are the mean of 2 independent experiments

\begin{tabular}{|c|c|c|c|c|c|}
\hline \multirow[b]{2}{*}{ Ingredient (g) } & \multicolumn{5}{|c|}{ Pentasodium triphosphate $(\%, \mathrm{wt} / \mathrm{wt})$} \\
\hline & 0 & 0.6 & 1.2 & 1.5 & 1.8 \\
\hline Micellar casein concentrate & 16.00 & 16.00 & 16.00 & 16.00 & 16.00 \\
\hline Sunflower oil & 16.00 & 16.00 & 16.00 & 16.00 & 16.00 \\
\hline Milli-Q water (ultrapure) $)^{1}$ & 48.00 & 48.00 & 48.00 & 48.00 & 48.00 \\
\hline Citric acid (monohydrate) ${ }^{2}$ & 0.34 & 0.43 & 0.50 & 0.55 & 0.59 \\
\hline Disodium phosphate (dihydrate) ${ }^{2}$ & 0.36 & 0.27 & 0.20 & 0.15 & 0.11 \\
\hline Pentasodium triphosphate & 0.00 & 0.50 & 1.00 & 1.25 & 1.50 \\
\hline \multicolumn{6}{|l|}{$\mathrm{pH}$} \\
\hline Premix & 5.91 & 5.92 & 5.94 & 5.91 & 5.88 \\
\hline Processed sample & 5.85 & 5.81 & 5.87 & 5.87 & 5.90 \\
\hline
\end{tabular}

${ }^{1}$ Merck KGaA.

${ }^{2}$ Used to adjust $\mathrm{pH}$ of processed sample (target $\mathrm{pH} 5.85 \pm 0.05$ ).

further concentrated (by a factor of 3.6) with the ceramic gradient membranes, cooled, and stored overnight at $4^{\circ} \mathrm{C}$. The following day, the concentrate was preheated to $40^{\circ} \mathrm{C}$ and then spray-dried in a co-current spray drier (Production Minor, GEA Niro) using a rotary disk at $15,000 \mathrm{rpm}$ at $190^{\circ} \mathrm{C}$ and $80^{\circ} \mathrm{C}$ inlet and outlet temperatures. The MCC powder was cooled, transferred to polyethylene bags, vacuum-sealed, and stored in the dark at ambient conditions until further use. Protein content was $85 \%$, with a casein-to-serum protein ratio of 98:2. Total calcium content was $1.69 \mathrm{~g} / 100 \mathrm{~g}$, which is comparable to values for commercial MCC powder as stated by other researchers (Cadesky et al., 2017). Sunflower oil was purchased from a local grocery store. Salts (PP; citric acid monohydrate, CA; and disodium phosphate, DSP) were of analytical grade and purchased from either Merck KGaA or Carl Roth GmbH. A mixture of CA-DSP was used to adjust $\mathrm{pH}(5.85 \pm$ $0.05)$ to exclude any effect on processed cheese functional properties due to $\mathrm{pH}$. The buffering salt ratio necessary to counter the $\mathrm{pH}$ increase was empirically determined for each level of PP. The amount added ( 0.7 $\mathrm{g}$ or $0.9 \% \mathrm{wt} / \mathrm{wt}$ ) was the minimum to maintain $\mathrm{pH}$ without affecting the viscosity curves, as determined in preliminary experiments (data not shown).

\section{Premix Preparation and Sample Processing}

Each of the 5 formulations was prepared and processed in duplicate. First, the salts were dissolved in Milli-Q water (Merck KGaA). Then, the sunflower oil was added and mixed using a dispersing instrument (T25 digital Ultra-Turrax, IKA Werke $\mathrm{GmbH}$ and Co. KG) at 10,000 rpm for $30 \mathrm{~s}$. Finally, the MCC powder was blended with the liquid phase using the same dispersing instrument at 10,000 rpm for $60 \mathrm{~s}$. The unprocessed mixture is referred to as "premix" to differentiate it from the sample after processing. The $\mathrm{pH}$ of both premix and processed samples was determined using a solid-state $\mathrm{pH}$ electrode (InLab Solids, Mettler Toledo).

Sample processing was performed in a rheometer (MCR 702, Anton Paar GmbH) with a custom-made geometry consisting of a cup with an inner diameter of $65.9 \mathrm{~mm}$, a solvent trap, a lid, and a 2-blade stirrer (Figure 1). Fifty grams of premix was transferred to the preheated geometry cup, the solvent trap was filled with $4 \mathrm{~mL}$ of demineralized water, the lid was closed, and the run was started. The premix was processed for 270 min with continuous agitation $(17.37 \mathrm{rpm})$ at $90^{\circ} \mathrm{C}$. The geometry, although invaluable to simultaneously process the cheese and follow the structure formation online, does not meet the conditions necessary to calculate absolute viscosities. Therefore, only torque values that arise from the flow resistance of the sample at the preset rotational speed are given and used as an
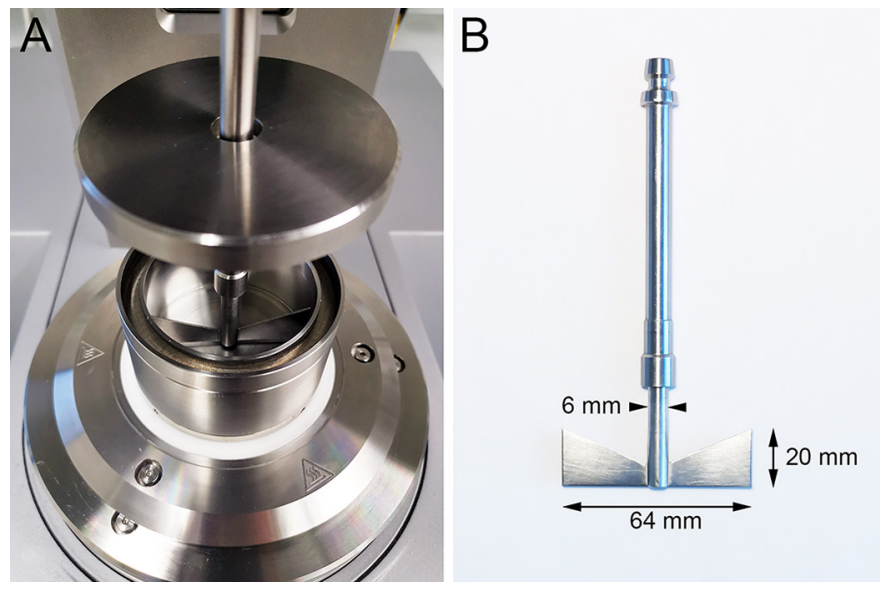

Figure 1. (A) Custom-made geometry with cup, solvent trap, lid, and 2-blade stirrer. (B) Close-up of the 2-blade stirrer with shaft and blade dimensions. 
indicator of apparent viscosity. Torque was measured every $15 \mathrm{~s}$.

\section{Determination of Textural Properties}

Hardness was determined in triplicate with a texture analyzer (TA.XTplus, Stable Micro Systems) equipped with a 500-g load cell. Samples ranged from viscous to solid, depending on temperature and formulation, thus requiring a customized setup and test procedure. Equal volumes of processed sample were poured into 3 custom-made hemispherical acrylic molds with a top diameter of $25 \mathrm{~mm}$ and a depth of $15 \mathrm{~mm}$. Samples were tempered at $20^{\circ} \mathrm{C}$ for $45 \mathrm{~min}$ before being centrally positioned below an acrylic cylinder probe with a diameter of $5 \mathrm{~mm}$. A uniaxial penetration test was then conducted (minimum contact force $1 \mathrm{mN}$, cross-head speed $0.5 \mathrm{~mm} / \mathrm{s}$, penetration depth $7.5 \mathrm{~mm}$ ). Hardness $(\mathrm{N})$ was defined as the maximum force of the compression (absolute peak force on the downstroke).

\section{Quantification of Insoluble Calcium by Acid-Base Titration}

The amount of insoluble calcium from processed cheese samples - that is, calcium from residual $\mathrm{CaP}$ nanoclusters that were sequestered by the phosphoserine clusters on the caseins (Lenton et al., 2020) - was determined via the acid-base titration method described in Wolfschoon-Pombo and Andlinger (2013), which is an adaptation of the standard titrimetric method by Hassan et al. (2004). Fifteen grams of processed sample and $120 \mathrm{~mL}$ of prewarmed $\left(50^{\circ} \mathrm{C}\right)$ sodium hydroxide $(3.3 \mathrm{mM})$ were mixed using the Ultra-Turrax dispersing instrument at 10,000 $\mathrm{rpm}$ for $2 \mathrm{~min}$. The mixture was cooled to $20^{\circ} \mathrm{C}$ in a water bath before adjustment to pH 7.0 with $0.5 \mathrm{~N}$ sodium hydroxide and stored overnight at $10^{\circ} \mathrm{C}$. The next morning, two $50-\mathrm{g}$ aliquots were taken from the mixture, filled into the prewarmed $\left(20^{\circ} \mathrm{C}\right)$ measurement beaker, and agitated at $400 \mathrm{rpm}$. Each aliquot was titrated from its initial $\mathrm{pH}$ to $\mathrm{pH}$ 3.0 with $0.5 \mathrm{~N}$ nitric acid and then from $\mathrm{pH} 3.0$ to 7.0 with $0.5 \mathrm{~N}$ sodium hydroxide. Titration was facilitated by the automated multichannel titrator (TitroWiCo, Cornelius Consult). The amount of insoluble calcium (insolCa) was calculated based on the addition of each titrant using the linear relationship shown in Equation [1] (Remillard and Britten, 2011):

$$
\text { insolCa }=72.25 \cdot \triangle A B,
$$

where $\triangle A B$ denotes the difference between the volumes of acid and base used in the $\mathrm{pH}$ range of 4.0 to 5.5.

\section{Quantification of Casein Fractions from Insoluble and Soluble Aggregates by RP-HPLC}

Reverse-phase (RP)-HPLC was performed as described in Dumpler et al. (2017). Five grams of processed sample and $15 \mathrm{~g}$ of Milli-Q water were mixed and homogenized first with a laboratory shaker (Vortex Genie 2, Bender and Hobein AG) for $1 \mathrm{~min}$ and then with a tube rotator (Fisherbrand Mini Tube Rotator, Fisher Scientific $\mathrm{GmbH}$ ) at $15 \mathrm{rpm}$ for $30 \mathrm{~min}$. For the quantification of casein fractions from large aggregates (found in the pellet) and small aggregates, or monomers, or both (found in the supernatant), 1,500 $\mu \mathrm{L}$ of the homogenized mixture were transferred to a $2-\mathrm{mL}$ microcentrifuge tube and centrifuged at maximum speed, 21,380 $\times \mathrm{g}$, for $10 \mathrm{~min}(\mathrm{Z} 233 \mathrm{M}-2$, HERMLE Labortechnik $\mathrm{GmbH})$. The resultant 3 phases were carefully separated, the fat layer was discarded, and $200 \mu \mathrm{L}$ of the supernatant were mixed with $800 \mu \mathrm{L}$ of guanidine buffer and analyzed via RP-HPLC, whereas the pellet $(0.075 \mathrm{~g})$ was mixed with $1,500 \mu \mathrm{L}$ guanidine buffer, stored overnight at $4^{\circ} \mathrm{C}$, brought to room temperature the next day, and centrifuged at $21,380 \times g$ for $10 \mathrm{~min}$. The collected supernatant was then used for RP-HPLC analysis. Each assay was run in duplicate.

\section{Light and Transmission Electron Microscopy}

When not otherwise stated, materials used for sample preparation were obtained from Electron Microscopy Sciences.

Sample Preparation. Immediately after processing was completed in the rheometer, a small sample of the hot mass was transferred to a glass Petri dish with a plastic spoon and spread to a thickness of approximately $3 \mathrm{~mm}$. The layer was very carefully flooded by slowly introducing fixative (formaldehyde and glutaraldehyde, $2.5 \%$ each, in $0.1 \mathrm{M}$ sodium cacodylate buffer, $\mathrm{pH}$ 7.4) from the side of the Petri dish until the sample was completely immersed. The covered sample rested for at least $2 \mathrm{~h}$ at room temperature before several strips of approximately $3 \times 3 \times 10 \mathrm{~mm}$ were cut with a sharp razorblade from the center of the sample and transferred to vials containing fresh fixative. A small piece of sponge, wetted with fixative solution, was carefully placed over the samples to ascertain complete immersion in fixative. Samples were stored at $4^{\circ} \mathrm{C}$ until all experiments were conducted. Samples were then shipped to the United States for further processing, as described in Vollmer et al. (2019).

Images of thin sections ( $\sim 500 \mathrm{~nm})$ were obtained using a digital optical microscope (VXH-5000, Keyence), and images of ultrathin sections $(\sim 70 \mathrm{~nm})$ were obtained using a transmission electron microscope (JEM 
1400 Plus, JEOL USA Inc.) equipped with a high-performance CCD camera (ORIUS SC1000, Gatan Inc.). Acceleration voltage was $120 \mathrm{kV}$.

Image Analysis and Image Processing. Three to five sections from 2 to 3 randomly selected polymerized blocks per replicate were analyzed, and images covering a wide range of magnifications (500 to $20,000 \times$ ) were taken from up to 10 fields of view. In total, over 1,300 images were recorded for this study, and the most representative images were selected for publication. When necessary, brightness and contrast levels of micrographs were adjusted in Adobe Photoshop 2021 (Adobe Inc.).

\section{Statistical Analysis}

Two rheological experiments per PP level were conducted and all analyses performed on samples resulting from those 10 independent runs were performed in either duplicate [insoluble calcium, RP-HPLC, light microscopy (LM), transmission electron microscopy (TEM)] or triplicate (cheese hardness). Average and standard deviation (SD) are reported where appropriate. Statistical significance of the mean values was evaluated by ANOVA, followed by either Tukey's or Dunnett's multiple comparisons test, at $P<0.05$, using Prism 9 (GraphPad Software Inc.).

\section{RESULTS AND DISCUSSION}

\section{Effect of Pentasodium Triphosphate Concentration on Apparent Viscosity}

As can be seen in Figure 2, torque values rapidly dropped within the first $10 \mathrm{~min}$ of processing for all tested concentrations of the PP emulsifying salt, which is assumed to be related to the time it took for the samples to reach the target temperature, as demonstrated by Kawasaki (2008). Compared with the control sample $(0 \% \mathrm{PP})$, all samples showed an increase in apparent viscosity (torque) with processing time, although the increase was only marginal (and not statistically significant) for the lowest concentration tested $(0.6 \% \mathrm{PP})$. This behavior has been noted by several investigators (Lee et al., 2003; Kawasaki, 2008; Heertje, 2014) and is commonly referred to as "creaming" or the "creaming reaction" in the processed cheese industry. It should be noted that the cheese samples contained $0.7 \mathrm{~g}$ of a varying mixture of CA and DSP. Although these salts were primarily used to maintain a cheese $\mathrm{pH}$ of $\sim 5.85$ during processing, they are also known to contribute to calcium chelation, albeit to a much lesser degree than PP. Importantly, the amount added $(0.9 \%)$ was not sufficient by itself to induce a viscosity increase.
Final torque values, at $270 \mathrm{~min}$ of processing (Table 2 ), depended on PP concentration and were higher for samples containing $1.2 \%, 1.5 \%$, and $1.8 \%$, compared with the control $(P<0.05)$. The kinetics of reaching these end points also differed, with torque values starting to increase earlier and faster for higher concentrations of PP (Figure 2). For samples containing 1.2\% PP and higher, they also started to fluctuate toward the end of processing, an indication that parts of the molten mass periodically adhered to the stirrer and were randomly released, thus making the corresponding torque measurements less accurate. We observed a similar behavior previously (Vollmer et al., 2021). Whether an increase in PP concentration also led to a shorter time for protein dispersal and solubilization was difficult to assess, as clear inflection points were not evident in the apparent viscosity profile. It is noteworthy that the initial torque values were higher for samples containing higher concentrations of $\mathrm{PP}$, which implies that the action of the emulsifying salts must have started already during the preparation of the premix, leading to differences in firmness even before processing in the rheometer commenced. It should be stated that the preparation of the premix followed a strict protocol and was accomplished within 6 to 7 min for all tested samples. Therefore, differences in the firmness of the premix were predominantly affected by $\mathrm{PP}$ concentration and not by handling conditions.

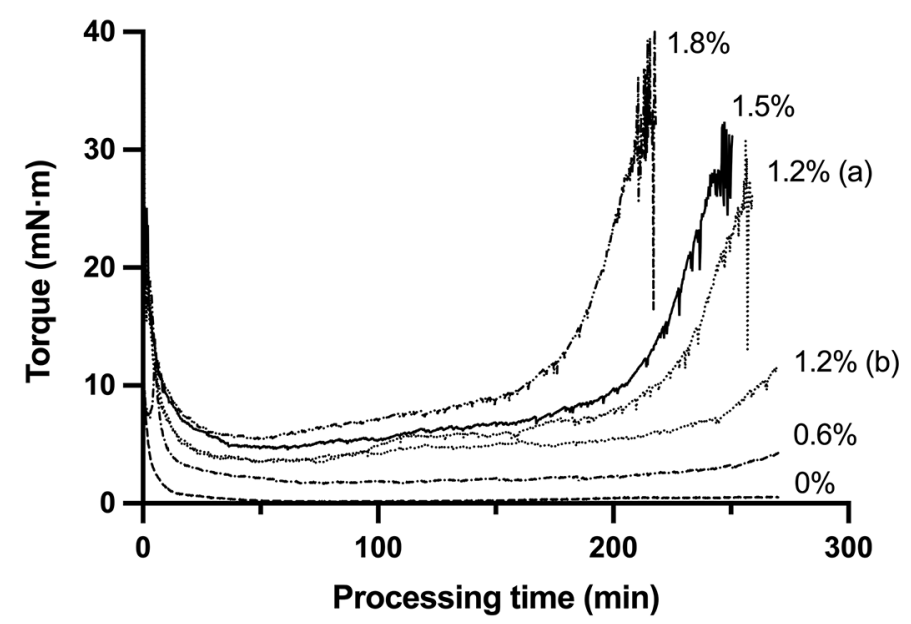

Figure 2. Torque values (indicating apparent viscosity) of model processed cheese as a function of pentasodium triphosphate (PP) concentration. Each experiment was run in duplicate, of which one representative curve is shown, with the exception of $1.2 \%$ PP. Here, curves from both independent experiments are shown [(a) and (b)] because they differed largely from each other at the end of the run. All samples were processed for $270 \mathrm{~min}$, but to improve clarity, curves for $1.2 \%$ (a), $1.5 \%$, and $1.8 \%$ are truncated at 259,250 , and $218 \mathrm{~min}$, respectively. 
Table 2. Effect of pentasodium triphosphate (PP) concentration on torque values (mean $\pm \mathrm{SD}, \mathrm{n}=2$ ) at the end of processing (270 min)

\begin{tabular}{lc}
\hline $\mathrm{PP}(\%, \mathrm{wt} / \mathrm{wt})$ & Torque $(\mathrm{mN} \cdot \mathrm{m})$ \\
\hline 0.0 & $0.39 \pm 0.19^{\mathrm{a}}$ \\
0.6 & $4.62 \pm 0.46^{\mathrm{a}}$ \\
1.2 & $20.04 \pm 11.62^{\mathrm{ab}}$ \\
1.5 & $32.39 \pm 2.70^{\mathrm{b}}$ \\
1.8 & $31.73 \pm 0.92^{\mathrm{b}}$ \\
\hline
\end{tabular}

${ }^{\mathrm{a}, \mathrm{b}}$ Mean values not sharing a common superscript are different $(P<$ $0.05)$.

The reproducibility of the viscosity curves was generally high; the 2 experiments containing $1.2 \% \mathrm{PP}$, however, were a notable exception and are therefore both included in Figure 2 [1.2\% (a) and 1.2\% (b)]. Although both curves still fall between the curves with $0.6 \%$ and $1.5 \%$, respectively, the large variability observed at this particular level of PP may indicate a critical point in the structure formation process that is sensitive to minimal perturbations in the system.

The increase in apparent viscosity along the processing time - that is, the "creaming reaction," or the "texturization reaction," as Vollmer et al. (2021) have recently suggested calling it - has been observed to occur in more or less distinct steps, depending on process and compositional parameters (Lenze et al., 2019; Vollmer et al., 2021), but no such steps were distinguishable in the current set of experiments. Rather, the viscosity increase was first gradual and then very rapid, particularly for higher concentrations of PP. Differences in sample formulation and stirring speed, and the design of the stirrer, might account for this different behavior.

\section{Effect of Pentasodium Triphosphate Concentration on Textural Properties}

Hardness values are shown in Figure 3. As was already evident from Figure 2 and Table 2, increasing the concentration of PP increased sample firmness. This was significant for concentrations of $1.2 \%$ and higher $(P$ $<0.05)$. The response appeared sigmoidal, which was confirmed by fitting a 4-parameter logistic curve $\left(\mathrm{R}^{2}\right.$ $=0.89$; data not shown). This suggests that we were approaching the maximum attainable firmness with $1.8 \%$ PP (plus 0.9\% CA-DSP) and, accordingly, using higher concentrations would not likely result in higher degrees of firmness, at least not under the conditions here tested. Not surprisingly, the SD for mean hardness of samples containing 1.2\% PP (plus $0.9 \%$ CA-DSP) was very large, mirroring the data from torque measurements.

In summary, the observed changes in cheese hardness suggest a structure formation process that was highly affected by the concentration of PP. The results by themselves were not unexpected, as similar data have been reported for other emulsifying salts (Shirashoji et al., 2006; Chen and Liu, 2012). In combination with the following analyses, however, we achieved a much better understanding of the intricate structure formation processes underlying the physicochemical behavior.

\section{Effect of Pentasodium Triphosphate Concentration on Insoluble Calcium}

Although the exact mechanism of action is not clear, it is known that emulsifying salts sequester or chelate calcium (Caric et al., 1985; Kapoor and Metzger, 2008). And because calcium is a major determinant of casein micelle stability as well as cheese texture (Fox et al., 2015), we quantified the insoluble calcium (calcium from residual $\mathrm{CaP}$ nanoclusters that was sequestered by the phosphoserine clusters on the caseins) in the processed cheese samples by acid-base titration. Results are presented in Figure 4. As anticipated, insoluble calcium levels decreased with increasing concentrations of PP $(P<0.05)$. Not only that, but this change was exponential. At a concentration of $1.2 \% \mathrm{PP}$, already $73 \%$ of insoluble calcium was chelated. Increasing the concentration to $1.8 \% \mathrm{PP}$ led to the removal of only an additional $12 \%$ of insoluble calcium from the casein matrix. Removal of more than $70 \%$ of $\mathrm{CaP}$ nanoclusters from milk samples has been reported to result in disintegration of the casein micelle (Fox et al., 2015). Based on these findings, a concentration of $1.2 \% \mathrm{PP}$ (plus $0.9 \%$ CA-DSP, for a total of $2.1 \%$ of emulsifying salts) constituted a critical threshold for the structure formation process in our system.

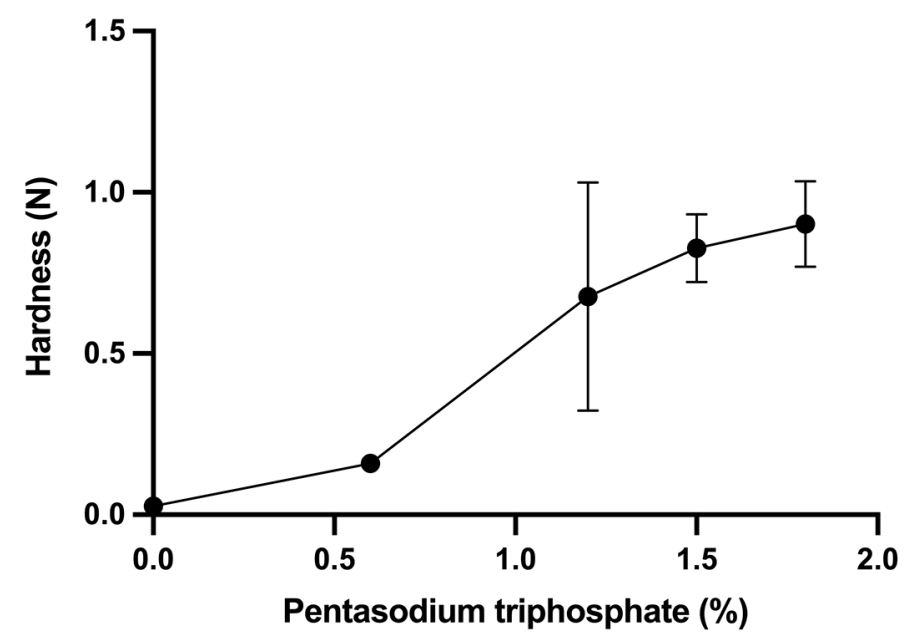

Figure 3. Hardness of model processed cheese samples after 270 min of processing, as a function of pentasodium triphosphate concentration. Mean and SD are shown from 2 independent experiments with 3 texture measurements each. 


\section{Effect of Pentasodium Triphosphate Concentration on Microstructure}

To visualize and better understand how calcium chelation by the emulsifying salts affected the structure formation, as well as how the textural properties fit in, processed cheese samples were analyzed first by LM and then by TEM. Figure 5 shows samples prepared for LM, in which proteins can be seen to have stained in shades of blue, whereas fat took on gray-greenish hues. As a control for the effect of the processing conditions (constant slow agitation over 270 min at $90^{\circ} \mathrm{C}$ ), the premix without any melting salt was analyzed first (Figure 5A). Here, the MCC powder was evident as spherical, ovoid, or irregularly shaped particles, often exceeding $100 \mu \mathrm{m}$ in size, although larger aggregates were also observed. Most particles appeared hollow, with the thickness of the wall varying. Interspersed between intact MCC powder particles were many, mostly small, fat globules $(<50 \mu \mathrm{m})$. When processed for $270 \mathrm{~min}$ with CA-DSP (used for $\mathrm{pH}$ adjustment) but without any PP, casein micelles were only partially dispersed, as evidenced by the overall light blue staining pattern of the matrix (Figure 5B). This was not unexpected, as it is known that complete rehydration and dispersal of spray-dried concentrated milk powders occurs over an extended time period depending on manufacturing, storage, and rehydration conditions (Anema et al., 2006; Baldwin and Truong, 2007; Crowley et al., 2015). The remaining MCC powder particles, or fragments thereof, were seen as compacted structures interspersed throughout the casein matrix (Figure 5B). Insufficient casein dispersal (and probably adsorption) caused coalescence of fat,

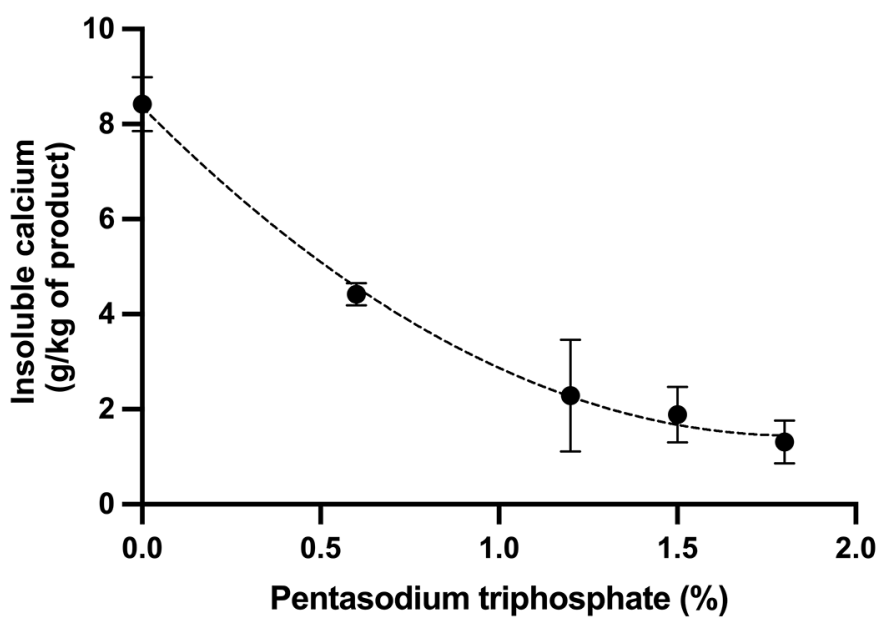

Figure 4. Insoluble (protein-bound) calcium in processed cheese samples as a function of pentasodium triphosphate concentration. Mean and SD are shown from 2 independent experiments with 2 titrations each. The dashed line indicates superimposed nonlinear trendline (quadratic fit, $\mathrm{R}^{2}=0.96$ ). as indicated by the presence of few, but very large, fat globules (some of them exceeding $100 \mu \mathrm{m}$ ), compared with the many small fat globules in the premix (Figure $5 \mathrm{~A})$. Adding $0.6 \% \mathrm{PP}$ to the system containing $0.9 \%$ CA-DSP, which together led to a reduction of insoluble calcium by $48 \%$ compared with $0 \% \mathrm{PP}$, completely changed the appearance of the fat globules; they were now so small and close to each other that they gave the impression of a uniform mass in which remnants of the casein micelle aggregates were embedded (Figure 5C). These remnants were all but gone in samples containing $1.2 \%$ PP (plus 0.9\% CA-DSP; Figure 5D), which makes this particular concentration appear critical; insoluble calcium was now reduced by $73 \%$. Chelating even more calcium $(78 \%$ and $85 \%)$ by increasing the PP concentration to $1.5 \%$ and $1.8 \%$, respectively, did not change the size of the fat globules noticeably (by qualitative assessment) but caused small areas without discernable internal structure to appear within the casein matrix (arrowheads in Figure 5E and F). These bore high resemblance to those observed in a comparable processed cheese model system containing $1.75 \% \mathrm{PP}, 0.88 \% \mathrm{CA}$, $0.44 \%$ DSP, and $0.44 \%$ trisodium citrate (Vollmer et al., 2021).

Analyzing the same samples with TEM at low instrumental magnification, as seen in Figure 6, helps to understand many of the changes described previously, and in some instances clarifies them. What had appeared in light micrographs of samples containing CA-DSP (but no PP) as whole MCC powder particles, or fragments thereof, were actually highly coagulated casein micelles, or proteinaceous material derived from those micelles, forming large aggregates. In some instances, the overall shape was very similar to that of intact powder particles, even possessing the typical central cavity (Figure $6 \mathrm{~B})$. However, with its densely reticulate pattern, the internal structure was markedly different than MCC powder particles, regardless of size. Although we cannot make any firm conclusions about the kinetics of this aggregation, as all micrographs were taken from samples that had been processed for $270 \mathrm{~min}$, it is likely that aggregate formation was fast in response to the high heat inside the rheometer cup $\left(90^{\circ} \mathrm{C}\right)$. If it had been a gradual process, we would not expect to see any aggregates that resembled intact MCC powder particles. Between these distinct aggregates were many smaller spherical structures with the appearance of typical casein micelles of milk; however, their larger size (often between $\sim 1$ and $2 \mu \mathrm{m}$ ) indicated that they too were most likely fusion products of individual casein micelles or fragments thereof. The lowest level of PP $(0.6 \%)$ was sufficient to cause complete dissociation of the small but not of the larger aggregates. Nevertheless, the compact structure of the large aggregates had taken on a loser, coral-like 
appearance, with fissures running deep into the interior from which protein material dissociated and filled adjacent areas (Figure 6C). Although LM analysis of samples containing $1.2 \% \mathrm{PP}$ indicated that the large, distinctive aggregates had completely disappeared (Figure 5D), TEM analysis demonstrated this not to be the case (Figure 6D). Instead, small regions of different electron density were observed within the casein matrix that are best described as unstructured, or amorphous. The higher the PP concentration in the system, the less electron-dense and more amorphous the structures were (Figure $6 \mathrm{D}-\mathrm{F}$ ). Although it would be natural to assume that these amorphous areas directly originated from coagulated casein micelles within the MCC powder particles as a consequence of the destabilizing effect of the emulsifying salts on the calcium-bridged protein network, we cannot make this conclusion, as we did not take samples during processing, only at the end, at 270 min. On the contrary, from experiments following the structure formation during the creaming reaction, we know that the MCC powder particles were completely hydrated, and nearly all casein micelles were dispersed and dissociated within 15 min of processing (when using $1.75 \% \mathrm{PP}$ ), after which the amorphous areas slowly appeared from a homogeneous casein matrix (Vollmer et al., 2021). Whether a similar behavior can be seen with lower concentrations of PP remains to be determined. Based on morphological similarities in samples with $1.2 \%$ and $1.5 \% \mathrm{PP}$, compared with samples with $1.8 \% \mathrm{PP}$ (Figure 5D-F and Figure 6D-F), it is not unreasonable to assume that $1.2 \% \mathrm{PP}$ (plus $0.9 \% \mathrm{CA}$ DSP) constitutes the minimum concentration for complete casein dissociation, with subsequent formation of the amorphous regions. A concentration of $0.6 \% \mathrm{PP}$ would most likely lead to only partial dissociation, due to insufficient calcium chelation to disrupt the majority of $\mathrm{CaP}$ nanoclusters within the casein network.

\section{Effect of Pentasodium Triphosphate Concentration on the Formation of Casein Fibrils}

The changes in the microstructure observed at higher magnification (referred to as ultrastructure) attest to an even more complex picture in response to increased PP concentrations (and thus decreased levels of protein-bound calcium). Figure 7 shows the contrasting changes inside and at the periphery of the distinctive casein aggregates, respectively, which are critical to our understanding of the reactions taking place in the processed cheese model system. From these high-magnifi-
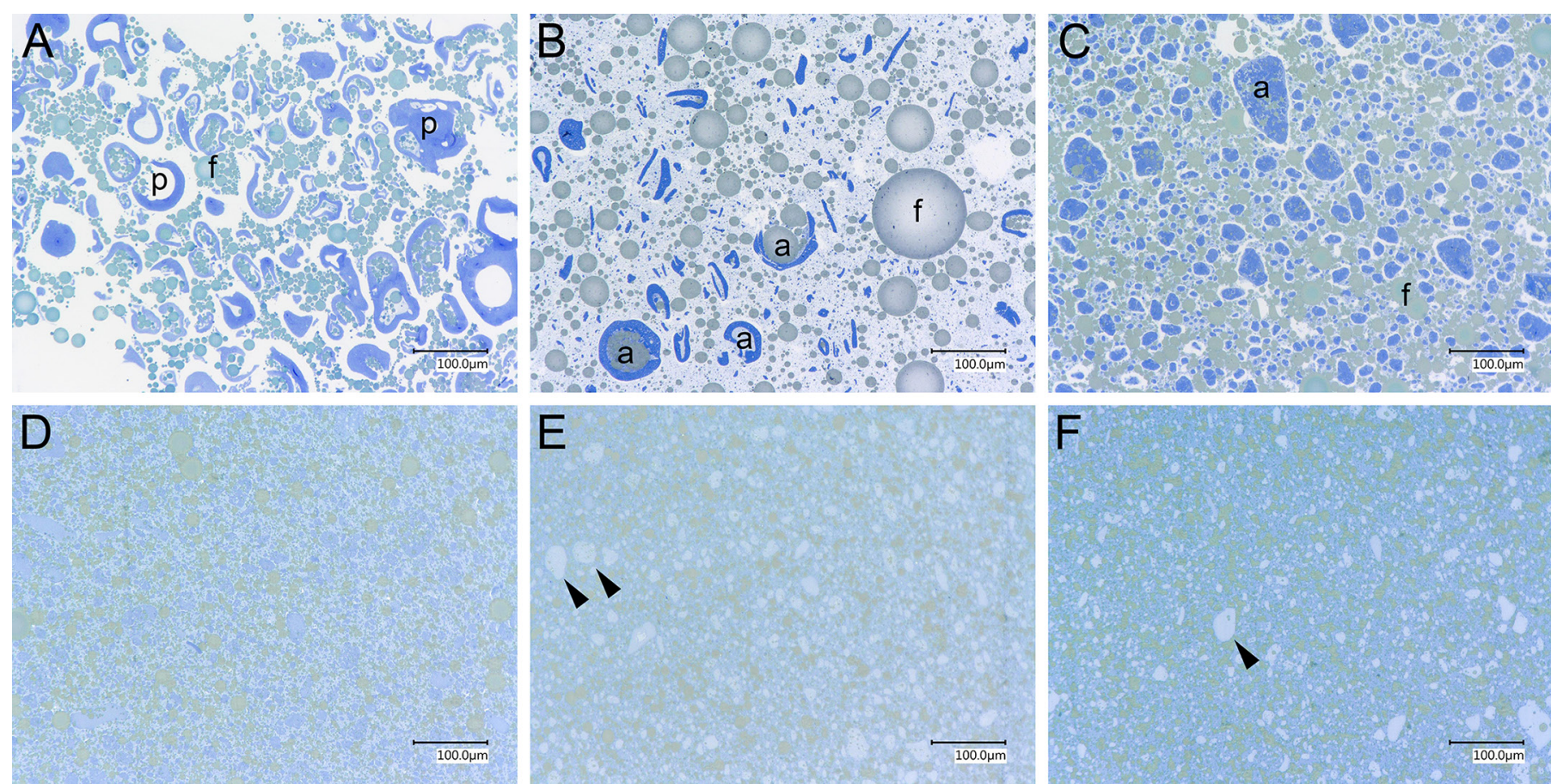

Figure 5. Changes in the microstructure of model processed cheese as a function of pentasodium triphosphate (PP) concentration. Light microscopy images of (A) premix without emulsifying salts and (B-F) samples containing increasing amounts of $\mathrm{PP}$ (plus $0.9 \%$ citric acid and disodium phosphate) after 270 min of processing: (B) $0 \%$, (C) $0.6 \%,(\mathrm{D}) 1.2 \%$, (E) $1.5 \%$, and (F) $1.8 \%$. Proteins are stained blue, and fat is stained gray-green. $\mathrm{a}=$ casein aggregate $\mathrm{f}=\mathrm{fat} ; \mathrm{p}=$ micellar casein concentrate powder particle. Arrowheads point to newly appearing amorphous areas within the casein matrix. Instrumental magnification at 500×, with the scale bar representing $100 \mu \mathrm{m}$. 

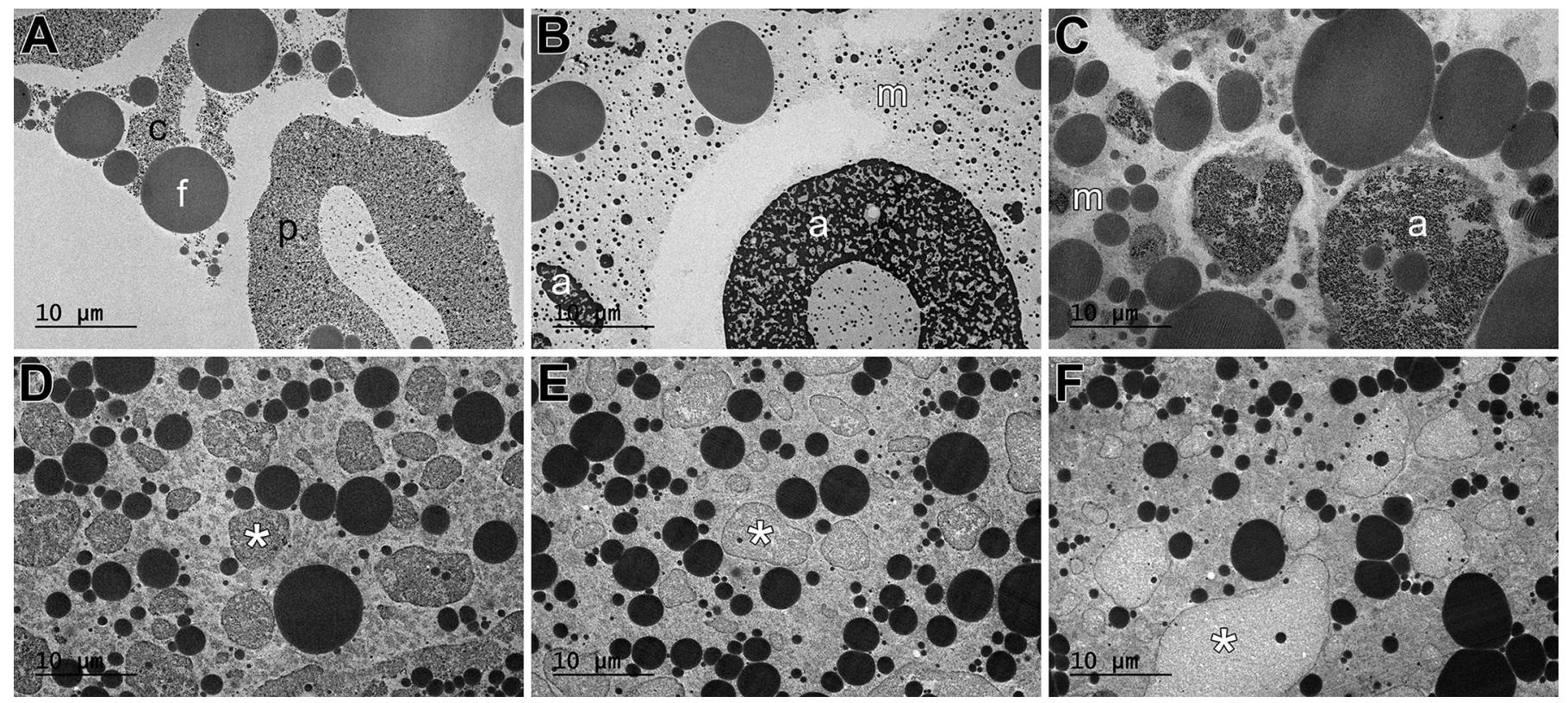

Figure 6. Changes in the microstructure of model processed cheese as a function of pentasodium triphosphate (PP) concentration. Transmission electron microscopy images of (A) premix without emulsifying salts and (B-F) samples containing various concentrations of $\mathrm{PP}$ (plus $0.9 \%$ citric acid and disodium phosphate) after 270 min of processing: (B) 0\%, (C) $0.6 \%,(\mathrm{D}) 1.2 \%,(\mathrm{E}) 1.5 \%$, and (F) $1.8 \%$. a = aggregated caseins; $\mathrm{f}=$ fat $; \mathrm{m}=$ casein matrix; $\mathrm{p}=$ micellar casein concentrate powder particle. Asterisks indicate newly appearing amorphous areas within the casein matrix. Instrumental magnification at $500 \times$, with the scale bar representing $10 \mu \mathrm{m}$.

cation micrographs it is apparent that shear, heat, and a small amount of ortho emulsifying salts was sufficient to cause fusion (or coagulation) of most of the spherical and tightly packed casein micelles into large, dense, and irregularly shaped aggregates, although some micelles had also dissociated and filled the spaces within and surrounding the aggregates with proteinaceous material. As more and more calcium was chelated, proteinprotein linkages that were mediated by calcium bridges within and between caseins were progressively destabilized, as reflected by prominent changes of the internal structure from densely reticulate to coral-like to particulate. As with results from other experiments in this study, a concentration of $1.2 \%$ PP (plus $0.9 \%$ CA-DSP) appeared to be critical, because higher concentrations changed the ultrastructure of the particulate material only slightly. This would be expected, as the combined salt concentration of $\sim 2.1 \%$ would bring the calcium chelating and hence emulsifying salt power to the level typically used for the manufacture of processed cheese.

From a structural point of view, the peripheral regions of the distinctive casein aggregates are of special interest. Here, an abrupt change was visible from the dense morphology of the aggregate into a randomly oriented network of short, irregular casein fibrils in samples containing $0.6 \%$ PP. When sectioned in longitudinal plane, the fibrils showed a distinctive dark-light-dark pattern, which corresponded to a circular structure with a hol- low core in cross-section. In 3 dimensions, this would translate into a tubular or rod shape with a central cavity. Fibrils were first exclusively observed at peripheral regions of casein aggregates, indicating a calcium chelating PP gradient. It appears that $0.6 \% \mathrm{PP}$ was not sufficient to disrupt the CaP linkages uniformly; that is, caseins were still tightly held together in the internal regions of the aggregates. With the chelation of more calcium ( $73 \%$ and higher), and thus breakage of $\mathrm{CaP}$ linkages in more internal regions as well, more protein was released from the casein aggregates, and fibril density in the casein matrix increased. Fibril morphology also changed, with fibrils becoming straighter. These data suggest that the action of the emulsifying salts not only destabilized the casein aggregates but led to a complete dissociation into the constituents of the bovine casein micelle $\left(\alpha_{\mathrm{S}^{-}} \mathrm{CN}, \alpha_{\mathrm{S}_{2}} \mathrm{CN}, \beta-\mathrm{CN}\right.$, and $\kappa-\mathrm{CN}$ ), of which one or more then participated in the formation of the fibrils, while the nonfibrillogenic caseins clustered together in separate amorphous regions. This behavior principally supports the proposed roles for caseins as agents of calcium sequestration and molecular chaperones preventing fibril formation (Carver et al., 2018).

We recently discovered very similar-appearing casein fibrils in a comparable processed cheese model system and concluded that the formation of these fibrils was at the heart of the so-called creaming reaction, or, as 
Nonfibrillogenic
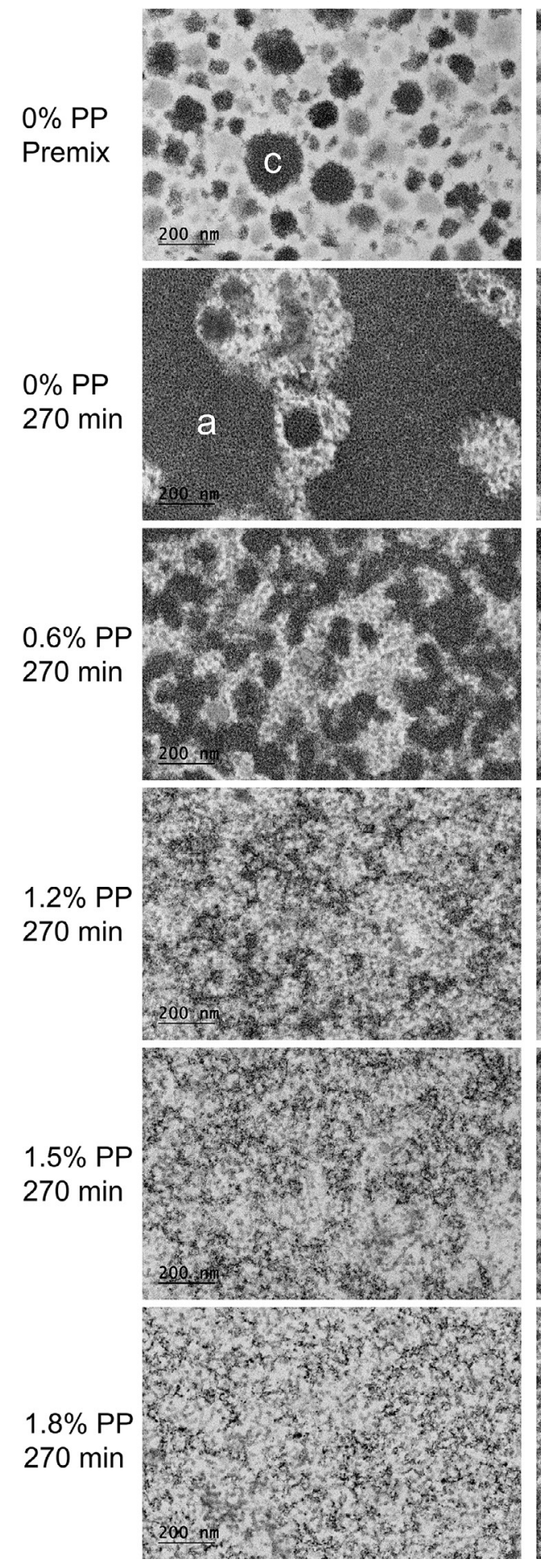

Fibrillogenic
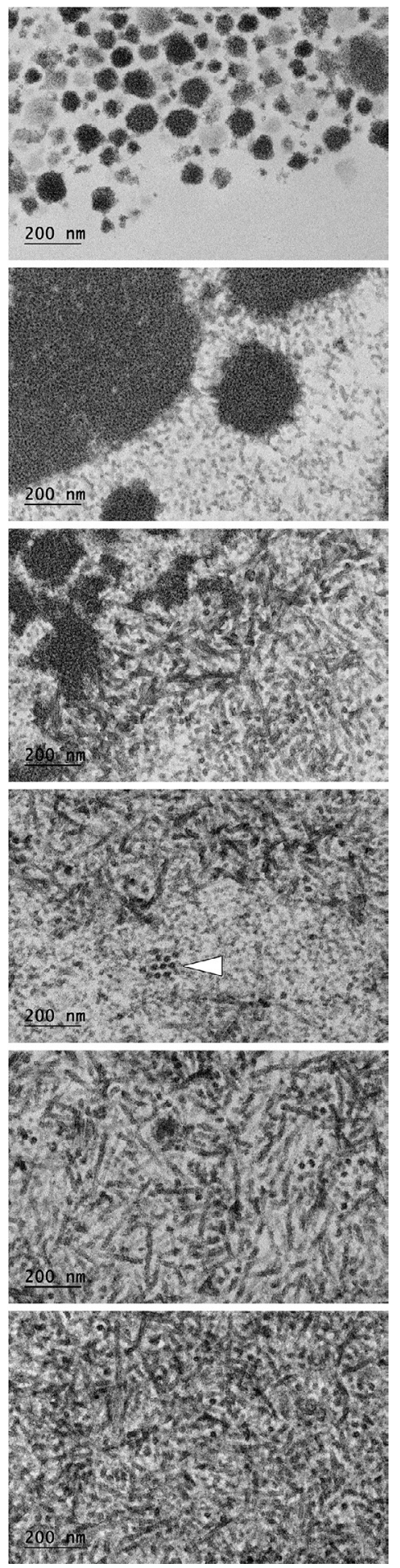

Figure 7. Development of amorphous and fibrillar regions within the casein matrix as a function of pentasodium triphosphate (PP) concentration. Transmission electron micrographs of thin sections from the premix without emulsifying salts and samples containing various concentrations of PP (plus $0.9 \%$ citric acid and disodium phosphate) after $270 \mathrm{~min}$ of processing. Micrographs in the left-hand column show the heat-induced aggregation of casein micelles, or of their dissociation products (0\% PP), partial loosening of the dense aggregate (0.6\% PP), and formation of amorphous areas (1.2\% to 1.8\%); micrographs in the right-hand column show the development of casein fibrils first observed at the periphery of the dense casein aggregates $(0.6 \% \mathrm{PP})$ and then increasingly accumulated within the casein matrix outside the amorphous areas $(1.2 \%$ to $1.8 \% \mathrm{PP})$. a = aggregated caseins; $\mathrm{c}=$ casein micelle. The arrowhead points to a small bundle of regularly aligned fibrils cut in the transverse plane. Instrumental magnification at 20,000×, with the scale bar representing $200 \mathrm{~nm}$. 
we proposed to call it, the texturization reaction, the gradual thickening of the molten cheese mass with prolonged processing (Vollmer et al., 2021). Others had also speculated that this was primarily a protein-based phenomenon involving some sort of protein reconstruction (Lee et al., 2003; Kawasaki, 2008; Heertje, 2014). In our previous study casein fibrils were typically longer (up to $1 \mu \mathrm{m}$ ), and many casein fibrils were furthermore seen in regularly aligned bundles at a similar processing time (275 min), but no such bundles were observed here, with the exception of only one small bundle, marked with an arrowhead in Figure 7 . It remains to be determined what conditions are amenable for fibril elongation and higher-order structuring to occur, but stir rate is an obvious candidate. It has been shown that stir rate affects the viscosity profile of processed cheese (Röck, 2010; Fu et al., 2018), although not always in the same fashion. Whereas an increase in the stir rate in the system used by Röck (2010) appeared to negatively affect the structure formation, the characteristic step-wise structure formation was only evident in the system used by Fu et al. (2018) after almost a 4-fold increase in stir speed.

Farrer and Lips (1999) also reported on rod-like aggregates in a $0.25 \%$ solution of sodium caseinate even in the absence of heat or shear. Sodium caseinate is produced from washed acid casein, followed by neutralization with alkali to $\mathrm{pH}$ 7.0. Hence, sodium caseinate has a much lower calcium content (by $\sim 10$ to $20 \times$ ) compared with rennet or micellar casein (O'Kennedy, 2011). A similarity between the fibrils observed by Farrer and Lips (1999) to those in our study would support the hypothesis that the calcium chelation activity of the emulsifying salts is central to fibril formation. Indeed, the fact that fibril formation was more pronounced and individual fibrils showed a more regular tubular appearance as higher concentrations of PP (including $0.9 \%$ CA-DSP) were used indicates that these protein aggregates were formed when casein proteins became completely devoid of bound CaP. However, separation in amorphous and fibrillar regions within the sodium caseinate solution was not visible, indicating that this might be particular to our processed cheese model system.

\section{Effect of Pentasodium Triphosphate Concentration on Emulsification}

The formation of a finer emulsion was already evident in LM images of processed samples (Figure 5), which showed fat globules to drastically decrease in size and to be more uniformly distributed. The analysis of the fat-protein interface with TEM explored the underlying mechanism and is presented in Figure 8. In the unheated premix, the surface of fat globules was virtually free of proteins (Figure 8A), and this changed only slightly in the control processed samples (Figure $8 \mathrm{~B}$ ). Addition of even small amounts of PP $(0.6 \%$, plus $0.9 \%$
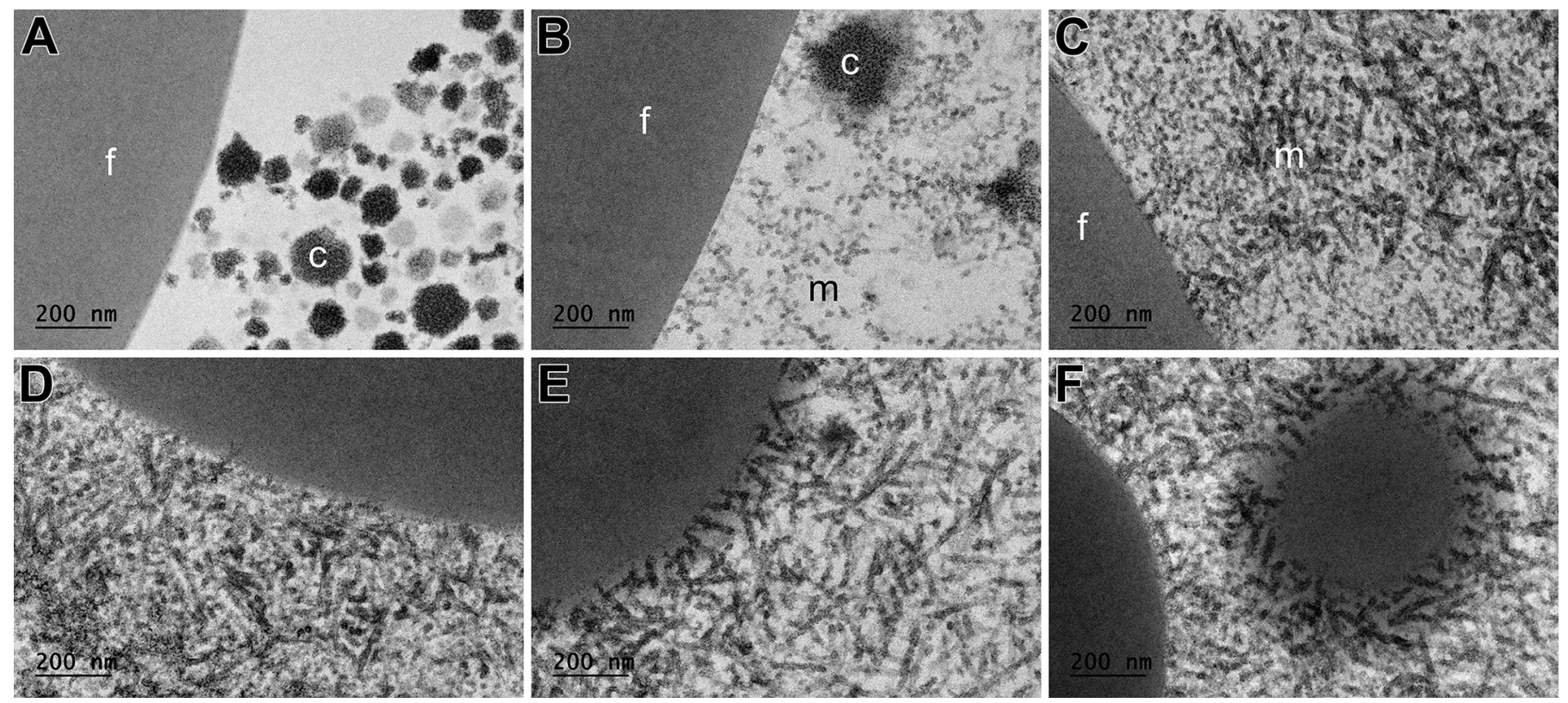

Figure 8. Adsorption of casein fibrils at the fat globule surface as a function of pentasodium triphosphate (PP) concentration. Transmission electron microscopy images of (A) premix without $\mathrm{PP}$ and $(\mathrm{B}-\mathrm{F})$ samples containing various concentrations of $\mathrm{PP}$ (plus $0.9 \%$ citric acid and disodium phosphate) after 270 min of processing: (B) $0 \%,(\mathrm{C}) 0.6 \%,(\mathrm{D}) 1.2 \%,(\mathrm{E}) 1.5 \%$, and (F) $1.8 \%$. $\mathrm{c}=$ casein micelle; $\mathrm{f}=$ fat; $\mathrm{m}=\mathrm{casein}$ matrix. Instrumental magnification at 20,000×, with the scale bar representing $200 \mathrm{~nm}$. 
CA-DSP) led to a noticeable protein adsorption (Figure $8 \mathrm{C}$ ), which was further enhanced at higher levels of PP (Figure 8D-F). As more and more casein fibrils formed within the casein matrix, they were progressively seen to attach to the fat globule surface. Because some casein fibrils also connected to each other, the strength of this network structure is assumed to have caused high local shear stress, which then led to the generation of smaller fat globules and therefore a finer emulsion.

Relating the observed microstructure (Figure 6) and ultrastructure (Figures 7 and 8) of the processed samples with their textural properties (Figures 2 and 3 ; Table 2), it becomes clear that the network first of casein particles and then of casein fibrils induced higher internal strength. However, firmness was initially low, as the network was not uniform and larger objects, such as the casein aggregates and their remnants, could still move relative to each other. Evidence of localized network disruption was visible in processed samples containing 0 and $0.6 \% \mathrm{PP}$ as electron-lucent "halos" around some of the larger casein aggregates (Figure 6B and $\mathrm{C}$ ). The investigated textural properties changed the most at a concentration of $1.2 \% \mathrm{PP}$, at which the small and localized networks of casein fibrils were observed to occupy larger areas as single, randomly oriented fibrils. We noticed some clear contact points between separate fibrils, but it is difficult to assess how many existed from 2-dimensional images. Higher PP concentrations led to an increase in fibril density, which translated into more attenuated effects on the textural properties.

\section{Effect of Pentasodium Triphosphate Concentration on Casein Fractions from Insoluble and Soluble Aggregates as a Means of Identifying Casein Fibril Constituents}

A central question of our present and previous studies (Vollmer et al., 2021) revolves around the nature of the casein fibrils. Even from high-magnification TEM it is not possible to identify the constituents of the fibrils based on morphology alone. Kawasaki (2008) demonstrated, by size-exclusion chromatography of water-soluble extracts from samples taken along the creaming reaction, that large aggregates of over 500 $\mathrm{kDa}$ (presumed to be casein clusters and which were not seen in the raw material) gradually disappeared with processing time. This coincided with an increase of a water-insoluble casein precipitate. Kawasaki (2008) speculated that the large casein clusters were reconstructed into a new insoluble casein network during the creaming reaction. Based on these findings, we hypothesized that the casein fibrils, as an integral part of the newly reconstructed casein network, would form an insoluble complex. In the following, we carried out centrifugation experiments to separate insoluble and soluble protein fractions and then identified the relative proportions of the individual casein species $\left(\alpha_{\mathrm{S} 1}-\mathrm{CN}\right.$, $\left.\alpha_{\mathrm{S}_{2}} \mathrm{CN}, \beta-\mathrm{CN}, \kappa-\mathrm{CN}\right)$ via $\mathrm{RP}-\mathrm{HPLC}$ to address the question of the fibril constituents.

As presented in Figure 9, RP-HPLC analysis of the pellet from processed control samples $(0 \% \mathrm{PP})$ showed a characteristic distribution of the 4 casein fractions with approximately $35 \% \quad \alpha_{\mathrm{S}^{-}} \mathrm{CN}, 15 \% \quad \alpha_{\mathrm{S}_{2}} \mathrm{CN}, 46 \%$ $\beta-\mathrm{CN}$, and $4 \% \kappa-\mathrm{CN}$. These values generally align with those found in the literature, where ratios of $4: 1: 4: 1.3$ have been reported (Walstra, 1990). In response to increasing $\mathrm{PP}$ concentrations, the $\kappa-\mathrm{CN}$ fraction most notably increased $(P=0.0299)$, whereas the $\beta-\mathrm{CN}$ fraction decreased. Values for $\alpha_{\mathrm{S}_{1}}-\mathrm{CN}$ and $\alpha_{\mathrm{S}_{2}} \mathrm{CN}$ fractions remained essentially the same. As expected, the ratio reversed in the supernatant, with a decrease in $\kappa$-CN $(P<0.0001)$ and an increase in $\beta-\mathrm{CN}(P=0.0031)$. These trends generally followed PP concentrations, with the exception of the highest concentration $(1.8 \%$ $\mathrm{PP}$ ). Several reasons might have contributed to this likely outlier. First, the error of the RP-HPLC method is $5 \%$, and because the presented data show fractions as a percentage of the total mass, a change in one of the casein fractions would invariably affect other fractions and their relative mass. Second, and more importantly, the system contained 3 phases after centrifugation (lipid layer, supernatant, pellet), but only the supernatant and pellet were further used for RP-HPLC. As Figure 8 conclusively shows, casein proteins progressively adsorbed to lipids and might therefore have been removed together with this layer. It is also important to consider that phase separation is based on density differences between the phases. Thus, with increased emulsification, smaller fat droplets with more protein adsorbed would have a much lover velocity and would not separate during centrifugation, therefore skewing the molar ratio. Consequently, values obtained for the sample containing 1.8\% PP were excluded from formal statistical analysis. For better comparison, the changes with their significance values are listed in Table 3.

Based on the significant increase of $\kappa-\mathrm{CN}$ in the pellet with concurrent significant decrease in the supernatant (and the opposite development for $\beta$-CN in the supernatant), it appears that, out of the 3 fibrillogenic candidates $\left(\alpha_{\mathrm{S}^{2}} \mathrm{CN}, \beta-\mathrm{CN}, \kappa-\mathrm{CN}\right.$; Farrell et al., 2003; Thorn et al., 2005; Thorn et al., 2008), $\kappa-\mathrm{CN}$ is the most likely constituent of the fibrils seen in this study. The diverging but complimentary trends in the distribution of $\kappa-\mathrm{CN}$ and $\beta-\mathrm{CN}$ in the pellet and supernatant, respectively, mirror the fibrillar and amorphous structure formation within the casein matrix and indicate a functional relationship where fibril formation is 

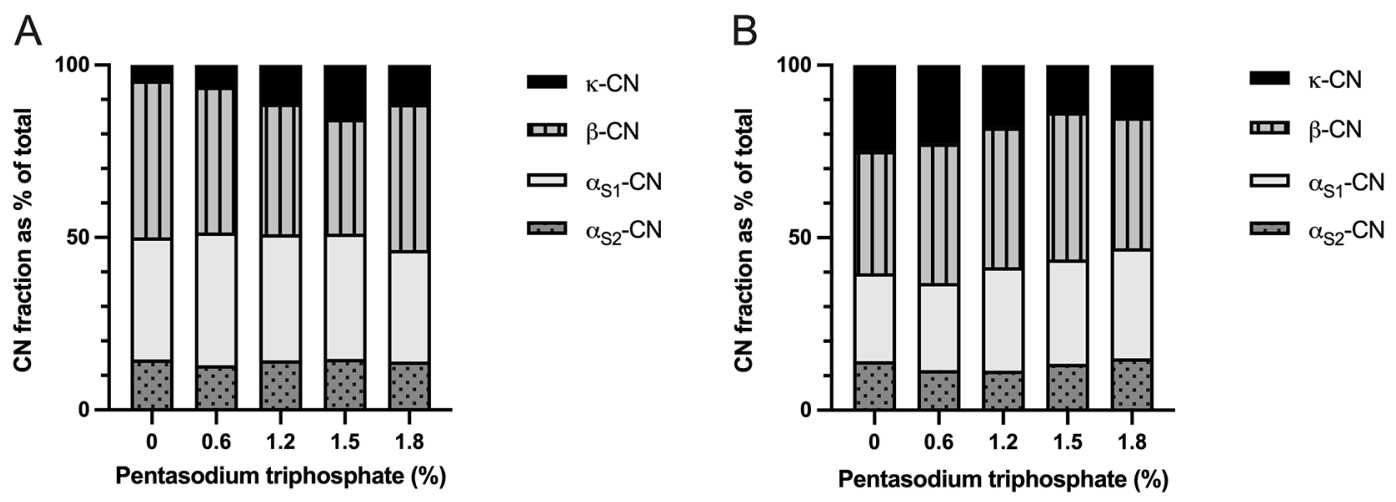

Figure 9. Relative casein mass fractions in the pellet (A) and in the supernatant (B) of centrifuged model processed cheese samples after 270 min of processing as a function of pentasodium triphosphate concentration. Means are shown of 2 independent experiments with 2 HPLC measurements each.

no longer suppressed by the chaperone activity of $\beta-\mathrm{CN}$ on its natural target, $\kappa-\mathrm{CN}$.

Because $\kappa-\mathrm{CN}$ is present in the system only at low proportions, yet the casein matrix of samples containing $1.2 \% \mathrm{PP}$ and higher showed a preponderance of casein fibrils in many areas, it is likely that another casein fraction was also involved. This is supported by the observation that casein fibril morphology gradually changed in response to increased PP concentrations, which coincided with a depletion of proteinaceous ma-

Table 3. Changes in relative $\mathrm{CN}$ fractions in the pellet and supernatant, respectively, of centrifugated model processed cheese samples after 270 min of processing, as a function of pentasodium triphosphate (PP) concentration; $\mathrm{n}=2$

\begin{tabular}{|c|c|c|}
\hline \multirow{2}{*}{$\begin{array}{l}\mathrm{CN} \text { fraction and } \\
\mathrm{PP} \text { level }(\%, w \mathrm{w} / \mathrm{wt})\end{array}$} & \multicolumn{2}{|c|}{$\begin{array}{l}\text { Direction of change } \\
\text { and significance level }^{1}\end{array}$} \\
\hline & Pellet & Supernatant \\
\hline \multicolumn{3}{|l|}{$\kappa-\mathrm{CN}$} \\
\hline 0.6 & NS & $\downarrow *$ \\
\hline 1.2 & NS & $\downarrow * * *$ \\
\hline 1.5 & $\uparrow *$ & $\downarrow * * * *$ \\
\hline \multicolumn{3}{|l|}{$\beta-\mathrm{CN}$} \\
\hline 0.6 & NS & $\uparrow * *$ \\
\hline 1.2 & NS & $\uparrow * *$ \\
\hline 1.5 & $\downarrow *$ & $\uparrow * *$ \\
\hline \multicolumn{3}{|l|}{$\alpha_{\mathrm{S} 1}-\mathrm{CN}$} \\
\hline 0.6 & $\uparrow *$ & NS \\
\hline 1.2 & NS & $\uparrow *$ \\
\hline 1.5 & NS & $\uparrow * *$ \\
\hline \multicolumn{3}{|l|}{$\alpha_{\mathrm{S} 2}-\mathrm{CN}$} \\
\hline 0.6 & NS & NS \\
\hline 1.2 & NS & NS \\
\hline 1.5 & NS & NS \\
\hline
\end{tabular}

$\overline{1 \uparrow}$ Increase, $\downarrow$ decrease, compared with control (0\% PP). NS = not statistically significant compared with control.

${ }^{*} P<0.05,{ }^{* *} P<0.01,{ }^{* * *} P<0.001,{ }^{*} * * * P<0.0001$, compared with control ( $0 \%$ PP), as analyzed by ANOVA followed by Dunnett's multiple comparisons test. terial from the amorphous areas. $\alpha_{S_{2}-} \mathrm{CN}$ might be a possible candidate, based on its in vitro capability of fibril formation. However, $\alpha_{\mathrm{S}_{2}} \mathrm{CN}$ amyloid fibril morphology is markedly different: $\alpha_{\mathrm{S}^{2}} \mathrm{CN}$ fibrils were described as twisted and ribbon-like, with the occasional loop formation (Thorn et al., 2008; Treweek et al., 2011; Thorn et al., 2014). Raynes et al. (2017) reported that when $\kappa-\mathrm{CN}$ and $\beta$-LG coaggregated, they formed fibrils that were morphologically different from their respective singular components. It is therefore conceivable that $\alpha_{\mathrm{S}_{2}}-\mathrm{CN}$ coaggregated with $\kappa-\mathrm{CN}$ to form fibrils that were also distinctively different from those seen for either component alone. No firm conclusion can be drawn for the effect of $\alpha_{\mathrm{S}_{1}}$-CN, as either no changes or only contradictory changes were observed (Table 3).

\section{CONCLUSIONS}

Increasing the concentration of PP had many diverse effects in our processed cheese model system that were all related to the chelation of protein-bound calcium and its impact on the microstructure. Without sufficient calcium to stabilize the casein protein network, amorphous and fibrillar regions were formed. The increasing network of casein fibrils is assumed to have higher internal strength, ultimately leading to higher apparent viscosity and cheese hardness and a finer emulsion. In essence, PP (including a small amount of CA and DSP), facilitated by its calcium chelation activity, induced a spatial separation of caseins with reported chaperone activity from their targets, thereby creating an environment that was amenable for the de novo genesis of these unique fibrils. Based on the progressive enrichment of $\kappa-\mathrm{CN}$ in insoluble fractions with concurrent losses from soluble fractions, fibrillogenic $\kappa-\mathrm{CN}$ is the most likely constituent of these fibrils, although probably not the only one. 


\section{ACKNOWLEDGMENTS}

Light microscopy was performed at the Utah Nanofab Laboratory, and electron microscopy was performed at the Health Sciences EM Core, both at the University of Utah, Salt Lake City. We thank Nancy Chandler (University of Utah) for technical assistance in sample preparation and Nabil Youssef (Utah State University) for valuable discussions and proofreading. Insightful and constructive comments by an anonymous reviewer are gratefully acknowledged. Project funding was available, in parts, through Deutsche Forschungsgemeinschaft (German Research Foundation, DFG), project no. 315464976, and the Research Foundation for Dairy Science at the Technical University of Munich, Germany. The authors have not stated any conflicts of interest.

\section{REFERENCES}

Anema, S. G., D. N. Pinder, R. J. Hunter, and Y. Hemar. 2006. Effects of storage temperature on the solubility of milk protein concentrate (MPC85). Food Hydrocoll. 20:386-393. https://doi.org/10 .1016/j.foodhyd.2005.03.015.

Baldwin, A. J., and G. N. T. Truong. 2007. Development of insolubility in dehydration of dairy milk powders. Food Bioprocess Technol. 85:202-208. https://doi.org/10.1205/fbp07008.

Brooker, B. E. 1978. The origin, structure and occurrence of corpora amylacea in the bovine mammary gland and in milk. Cell Tissue Res. 191:525-538. https://doi.org/10.1007/BF00219815.

Cadesky, L., M. Walkling-Ribeiro, K. T. Kriner, M. V. Karwe, and C. I. Moraru. 2017. Structural changes induced by high-pressure processing in micellar casein and milk protein concentrates. J. Dairy Sci. 100:7055-7070. https://doi.org/10.3168/jds.2016-12072.

Caric, M., M. Gantar, and M. Kalab. 1985. Effects of emulsifying agents on the microstructure and other characteristics of process cheese-A review. Food Microstruct. 4:297-312.

Carver, J. A., H. Ecroyd, R. J. W. Truscott, D. C. Thorn, and C. Holt. 2018. Proteostasis and the regulation of intra- and extracellular protein aggregation by ATP-independent molecular chaperones: Lens $\alpha$-crystallins and milk caseins. Acc. Chem. Res. 51:745-752. https://doi.org/10.1021/acs.accounts.7b00250.

Carver, J. A., and C. Holt. 2019. Chapter 5: Functional and dysfunctional folding, association and aggregation of casein. Pages 163-216 in Protein Misfolding. R. Donev, ed. Elsevier Inc. https:/ /doi.org/10.1016/bs.apcsb.2019.09.002.

Chen, L., and H. Liu. 2012. Effect of emulsifying salts on the physicochemical properties of processed cheese made from mozzarella. J. Dairy Sci. 95:4823-4830. https://doi.org/10.3168/jds.2012-5480.

Claudon, C., M. Francin, E. Marchal, J. Straczeck, F. Laurent, and P. Nabet. 1998. Proteic composition of corpora amylacea in the bovine mammary gland. Tissue Cell 30:589-595. https://doi.org/ 10.1016/S0040-8166(98)80040-2.

Crowley, S. V., B. Desautel, I. Gazi, A. L. Kelly, T. Huppertz, and J. A. O'Mahony. 2015. Rehydration characteristics of milk protein concentrate powders. J. Food Eng. 149:105-113. https://doi.org/ 10.1016/j.jfoodeng.2014.09.033.

Dumpler, J. 2018. Heat Stability of Concentrated Milk Systems: Kinetics of the Dissociation and Aggregation in High Heated Concentrated Milk Systems. p. 146. Springer Spektrum.

Dumpler, J., H. Wohlschläger, and U. Kulozik. 2017. Dissociation and coagulation of caseins and whey proteins in concentrated skim milk heated by direct steam injection. Dairy Sci. Technol. 96:807826. https://doi.org/10.1007/s13594-016-0304-3.

Farrell, H. M. Jr., P. H. Cooke, E. D. Wickham, E. G. Piotrowski, and P. D. Hoagland. 2003. Environmental influences on bovine к-casein: Reduction and conversion to fibrillar (amyloid) structures. J. Protein Chem. 22:259-273. https://doi.org/10.1023/A 1025020503769.

Farrer, D., and A. Lips. 1999. On the self-assembly of sodium caseinate. Int. Dairy J. 9:281-286. https://doi.org/10.1016/S0958 $-6946(99) 00075-8$

Fox, P. F., T. Uniacke-Lowe, P. L. H. Mesweeney, and J. A. O'Mahony. 2015. Dairy Chemistry and Biochemistry. Springer International Publishing AG.

Fu, W., Y. Watanabe, H. Satoh, K. Inoue, N. Moriguchi, K. Fusa, Y. Yanagisawa, T. Mutoh, and T. Nakamura. 2018. Effects of emulsifying conditions on creaming effect, mechanical properties and microstructure of process cheese using a rapid visco-analyzer. Biosci. Biotechnol. Biochem. 82:476-483. https://doi.org/10.1080/ 09168451.2018.1431106.

Hassan, A., M. E. Johnson, and J. A. Lucey. 2004. Changes in the proportions of soluble and insoluble calcium during the ripening of cheddar cheese. J. Dairy Sci. 87:854-862. https://doi.org/10.3168/ jds.S0022-0302(04)73229-4.

Heertje, I. 2014. Structure and function of food products: A review. Food Struct. 1:3-23. https://doi.org/10.1016/j.foostr.2013.06.001.

Kapoor, R., and L. E. Metzger. 2008. Process cheese: Scientific and technological aspects - A review. Compr. Rev. Food Sci. Food Saf. 7:194-214. https://doi.org/10.1111/j.1541-4337.2008.00040.x.

Kawasaki, Y. 2008. Influence of "creaming" on the properties of processed cheese and changes in the structure of casein during cheese making. Milchwissenschaft 63:149-152.

Kimura, T., and S. Taneya. 1975. Electron microscopic observation of casein particle in cheese. J. Electron Microsc. (Tokyo) 24:115-117. https://doi.org/10.1093/oxfordjournals.jmicro.a049955.

Lee, S. K., R. J. Buwalda, S. R. Euston, E. A. Foegeding, and A. B. McKenna. 2003. Changes in the rheology and microstructure of processed cheese during cooking. Lebensm. Wiss. Technol. 36:339345. https://doi.org/10.1016/S0023-6438(03)00012-4.

Lencki, R. W. 2007. Evidence for fibril-like structure in bovine casein micelles. J. Dairy Sci. 90:75-89. https://doi.org/10.3168/jds.S0022 -0302(07)72610-3.

Lenton, S., Q. Wang, T. Nylander, S. Teixeira, and C. Holt. 2020 Structural biology of calcium phosphate nanoclusters sequestered by phosphoproteins. Crystals (Basel) 10:755. https://doi.org/10 $.3390 /$ cryst10090755.

Lenze, S., A. Wolfschoon-Pombo, K. Schrader, and U. Kulozik. 2019. Effect of compositional factors and processing conditions on the creaming reaction during process cheese manufacturing. Food Bioprocess Technol. 12:575-586. https://doi.org/10.1007/s11947-019 $-2234-6$.

Leonil, J., G. Henry, D. Jouanneau, M. Delage, V. Forge, and J. L. Putaux. 2008. Kinetics of fibril formation of bovine k-casein indicate a conformational rearrangement as a critical step in the process. J. Mol. Biol. 381:1267-1280. https://doi.org/10.1016/j .jmb.2008.06.064.

Masotti, F., S. Cattaneo, M. Stuknyte, and I. De Noni. 2018. Status and development in analogue cheese formulations and functionalities. Trends Food Sci. Technol. 74:158-169. https://doi.org/10 $.1016 /$ j.tifs.2018.02.016.

Mizuno, R., and J. A. Lucey. 2005. Effects of two types of emulsifying salts on the functionality of nonfat pasta filata cheese. J. Dairy Sci. 88:3411-3425. https://doi.org/10.3168/jds.S0022-0302(05)73025 -3 .

O'Kennedy, B. T. 2011. Caseins. Pages 13-29 in Handbook of Food Proteins. G. O. Philipps and P. A. Williams, ed. Woodhead Publishing.

Pan, K., and Q. Zhong. 2015. Amyloid-like fibrils formed from intrinsically disordered caseins: Physicochemical and nanomechanical properties. Soft Matter 11:5898-5904. https://doi.org/10.1039/ C5SM01037C

Raynes, J. K., L. Day, P. Crepin, M. H. Horrocks, and J. A. Carver. 2017. Coaggregation of $\kappa$-casein and $\beta$-lactoglobulin produces morphologically distinct amyloid fibrils. Small 13:1603591. https://doi .org/10.1002/smll.201603591. 
Remillard, N., and M. Britten. 2011. Quantitative determination of micellar calcium in milk and cheese using acid-base titration. Milchwissenschaft 66:137-140.

Röck, S. M. 2010. Untersuchungen von Strukturbildungsvorgängen in komplexen Milchsystemen - dargestellt am Beispiel von Schmelzkäse [Investigation of structure formation in complex dairy systems-Analyzed using processed cheese as an example]. Dissertation. Technical University Munich, Germany.

Shirashoji, N., J. J. Jaeggi, and J. A. Lucey. 2006. Effect of trisodium citrate concentration and cooking time on the physiochemical properties of pasteurized process cheese. J. Dairy Sci. 89:15-28. https://doi.org/10.3168/jds.S0022-0302(06)72065-3.

Tamime, A. Y., M. Kalab, G. Davies, and M. F. Younis. 1990. Microstructure and firmness of processed cheese manufactured from cheddar cheese and skim milk powder cheese base. Food Struct. 9:23-37.

Taneya, S., T. Kimura, T. Izutsu, and W. Buchheim. 1980. The submicroscopic structure of processed cheese with different melting properties. Milchwissenschaft 35:479-481.

Thorn, D. C., H. Ecroyd, and J. A. Carver. 2014. Chapter 30: Polymorphism in casein protein aggregation and amyloid fibril formation. Pages 323-331 in Bio-Nanoimaging: Protein Misfolding and Aggregation. V. N. Uversky and Y. L. Lyubchenko, ed. Academic Press. https://doi.org/10.1016/B978-0-12-394431-3.00030-4.

Thorn, D. C., H. Ecroyd, M. Sunde, S. Poon, and J. A. Carver. 2008. Amyloid fibril formation by bovine milk $\alpha_{\mathrm{S}_{2}}$-casein occurs under physiological conditions yet is prevented by its natural counterpart, $\alpha_{\mathrm{S} 1}$-casein. Biochemistry 47:3926-3936. https://doi.org/10 $.1021 /$ bi701278c.

Thorn, D. C., S. Meehan, M. Sunde, A. Rekas, S. L. Gras, C. E. MacPhee, C. M. Dobson, M. R. Wilson, and J. A. Carver. 2005. Amyloid fibril formation by bovine milk k-casein and its inhibi- tion by the molecular chaperones $\alpha_{S^{-}}$and $\beta$-casein. Biochemistry 44:17027-17036. https://doi.org/10.1021/bi051352r.

Treweek, T. M., D. C. Thorn, W. E. Price, and J. A. Carver. 2011. The chaperone action of bovine milk $\alpha_{\mathrm{S}^{-}}$and $\alpha_{\mathrm{S}^{2}}$-caseins and their associated form $\alpha_{S}$-casein. Arch. Biochem. Biophys. 510:42-52. https://doi.org/10.1016/j.abb.2011.03.012.

Vollmer, A. H., I. Kieferle, N. N. Youssef, and U. Kulozik. 2021. Mechanisms of structure formation underlying the creaming reaction in a processed cheese model system as revealed by light and transmission electron microscopy. J. Dairy Sci. https://doi.org/10.3168/jds .2020-20080.

Vollmer, A. H., N. N. Youssef, J. A. Powell, and D. J. McMahon. 2019. Progressive ultrastructural changes in the casein matrix during the ripening of inadequately acidified feta cheese. J. Dairy Sci. 102:7734-7746. https://doi.org/10.3168/jds.2019-16395.

Walstra, P. 1990. On the stability of casein micelles. J. Dairy Sci. 73:1965-1979. https://doi.org/10.3168/jds.S0022-0302(90)78875 $-3$.

Wolfschoon-Pombo, A., and D. J. Andlinger. 2013. Micellar calcium in acid curd cheese with different $\mathrm{pH}$ values, fat and protein levels. Int. Dairy J. 32:20-25. https://doi.org/10.1016/j.idairyj.2013 .03 .011 .

Zehren, V. L., and D. D. Nussbaum. 1992. Process Cheese. Cheese Reporter Publishing Company, Green Bay, WI.

\section{ORCIDS}

Almut H. Vollmer @ https://orcid.org/0000-0001-5647-6524

Ingrun Kieferle (1) https://orcid.org/0000-0002-2776-0072

Ulrich Kulozik @ i https://orcid.org/0000-0001-9598-9242 